\title{
Original Research Article on Study of Correlation between Various Clinical Parameters and Hypoxemia in Children with Lower Respiratory Tract Infection of Age 2 Months to 5 Years
}

\author{
Chinmay Sahu', Deshish Kumar Panda ${ }^{2}$, Surjit Naik ${ }^{3}$, Saiprasanna Behera ${ }^{4}$ \\ ${ }^{1}$ Senior Resident, ${ }^{2,3}$ Asst. Prof in Paediatrics, Santha Bhima Bhoi Medical College \& Hospital, \\ Balangir. Odisha - 767001 \\ ${ }^{4}$ Research Associate, CRO Pharmatrendz Private Limited, Bhubaneswar, Odisha-751014
}

Corresponding Author: Saiprasanna Behera

\begin{abstract}
A case control study was conducted from October 2018 to September 2020 by taking children between the age group 2 months to 5 years, admitted to Sishu Bhawan and SCB Medical College Hospital, Cuttack with an acute history of cough and rapid respiration or difficulty in breathing, to know the correlation between various clinical parameters and hypoxemia along with ability of each parameter to predict the presence of hypoxemia in children. Among 109 children, 50 children were hypoxemic and 59 children were non hypoxemic with the prevalence of hypoxemia about $45.8 \%$. Among symptoms in children with Acute Lower Respiratory Tract Infection (ALRI), inability to feed, cough and difficulty in breathing had a sensitivity of $100 \%, 98 \%$ and $92 \%$ respectively and specificity was maximum for difficulty in breathing (62.7\%) to predict hypoxemia. On comparison between hypoxemic and non-hypoxemic children difficulty in breathing and inability to feed had a $\mathrm{p}$ value $<0.001$ and was statistically significant. Among signs in children with ALRI, appearance of the child, lower chest retraction, respiratory rate had a sensitivity of 100\%, $100 \%$ and $98 \%$ respectively to predict hypoxemia. Central cyanosis was the most specific sign with the specificity of $100 \%$ to predict hypoxemia followed by grunting with a specificity of $96.6 \%$. When compared between hypoxemic and non-hypoxemic children the p-value was very highly significant for appearance of nasal
\end{abstract}

flaring, grunting, suprasternal indrawing parameters whereas upon combination of variables, a combination of cough, inability to feed, tachypnoea and the combination of cough, subcostal retractions, tachypnoea had high sensitivity of $96 \%$ and $95 \%$ respectively to predict hypoxemia in children with ALRI. Combination of difficulty in breathing, cyanosis and grunting had high specificity of $98 \%$. Keeping in view of less feasibility of detection of hypoxemia in lower respiratory tract infection by pulse oximeter and $\mathrm{ABG}$ in developing countries and no available study in the state of Odisha, this study is conducted in an attempt to find out the alternative methods by various clinical parameters to detect hypoxemia which are easily recognized by the health personnel in developing countries for the further management of Lower Respiratory Tract Infection (LRTI).

Key words: Hypoxemia, Acute Lower Respiratory Tract Infection, Lower Respiratory Tract Infection, Management

\section{INTRODUCTION}

Acute respiratory infection is defined as acute onset of respiratory symptoms including cough, rhinorrhoea, fast/difficulty in breathing, chest wall indrawing and wheeze of less than 14 days duration1. Acute lower respiratory tract infection (ALRI) are the major cause of morbidity and mortality among children in 
developing countries, accounting for about $30 \%$ of mortality in children under 5 years of age $\mathrm{e}^{1,2,3}$. ALRIs are important indicators of the health disparities that persist between indigenous and non-indigenous children in developed countries.

Hypoxemia is defined as oxygen saturation less than $90 \%^{1,2,3,4}$ and is an important risk factor for mortality in children with ALRI . The most reliable way to detect hypoxemia is an arterial blood gas analysis or the determination of arterial haemoglobin saturation by pulse oximeter ${ }^{1,2,3,4,5}$.

Detection of hypoxemia by use of pulse oximetry and ABG analysis is not feasible in most situations in developing countries. Therefore it is important to accurately identify hypoxemia in children by use of clinical signs alone. Various symptoms and signs such as cough, difficulty breathing, cyanosis, grunting, subcostal retractions, sick appearance etc. have been evaluated for their ability to predict hypoxemia ${ }^{1,2,3}$.

\section{MATERIALS AND METHODS}

A case control study was conducted from October 2018 to September 2020 by taking children between the age group 2 months to 5 years, admitted to Sishu Bhawan and SCB Medical College Hospital, Cuttack with an acute history of cough and rapid respiration or difficulty in breathing.

Sampling Method: Purposive sampling

Inclusion Criteria: Children between the age of 2 months- 5 years presenting with an acute history of cough and rapid respiration or difficulty in breathing were included in the study according to the WHO criteria for ALRI.

Exclusion Criteria: Children presenting with ALRI along with the following condition:-

1. Diarrhoea

2. Convulsion

3. Known case of Asthma

4. Congenital heart disease
5. Severe anaemia

6. Shock

7. Severe dehydration.

8. Requiring ventilator support.

\section{METHODOLOGY}

109 children between the age group of 2 months to 5 years with history of cough and rapid respiration or breathing difficulty admitted to Sishu Bhawan and SCB College Hospital fulfilling the inclusion criteria were included in the study.

At the time of enrolment an informed written consent was obtained from the parents.

The study sample was divided into 2 groups. Group-1; children having oxygen saturation $<90 \%(\mathrm{n}=50)$. Group-2; children having saturation $>90$ on admission $(n=59)$. Baseline characteristics were compared. Frequency of different symptoms/ signs in both groups was calculated.

A history was obtained from the parents about the presence and duration of various symptoms: Cough, fever, difficulty in breathing, cry, inability to drink/feed. The child was examined and the followings signs were recorded. Appearance, heart rate, respiratory rate (counted for 60 seconds when the child is quite and at rest), cyanosis, grunting, nasal flaring, suprasternal indrawing, lower chest retractions, intercostal retraction, crepitation, rhonchi and decreased air entry.

A portable oximeter was used to measure oxygen saturation with an appropriate sized sensor on the finger or the toe while the child was breathing at room air. Hypoxemia was defined as oxygen saturation $<90 \%$.

\section{Analysis}

Data was collected by proper physical examination. Appropriate tables and graphical representations were used to display the data. Statistical significance was evaluated using the Chi square test. "p" value was obtained and a value $<0.05$ was taken as significant. 


\section{DISCUSSION}

In the case control study conducted from October 2018 to September 2020 by taking 109 children between the age group 2 months to 5 years, admitted to Sishu Bhawan and SCB Medical College Hospital, Cuttack with an acute history of cough and rapid respiration or difficulty in breathing, 50 children were found hypoxemic and 59 children were non hypoxemic with the prevalence of hypoxemia about $45.8 \%$. Among symptoms in children with lower respiratory tract infections inability to feed and cough had a good sensitivity of $100 \%$ and $98 \%$ to predict hypoxemia and none of the symptoms were specific to predict hypoxemia.

Table 1: Frequency of symptoms associated with hypoxemia

\begin{tabular}{|l|c|c|c|c|c|c|c|}
\hline \multicolumn{1}{|c|}{ Symptoms } & $\begin{array}{c}\text { Hypoxemic } \\
\text { children (50) }\end{array}$ & $\begin{array}{c}\text { Non-hypoxemic } \\
\text { children (59) }\end{array}$ & Sensitivity & Specificity & Pvalue & PPV & NPV \\
\hline Cough & 49 & 58 & $98 \%$ & $1.7 \%$ & 0.096 & $46 \%$ & $50 \%$ \\
\hline Fever & 39 & 41 & $78 \%$ & $30.5 \%$ & 0.316 & $49 \%$ & $62 \%$ \\
\hline Difficultyinbreathing & 46 & 22 & $92 \%$ & $62.7 \%$ & $<0.001$ & $68 \%$ & $90 \%$ \\
\hline Inabilityto Feed & 50 & 48 & $100 \%$ & $18.6 \%$ & $<0.001$ & $51 \%$ & $100 \%$ \\
\hline
\end{tabular}

Among the symptoms, inability to feed, cough and difficulty in breathing had high sensitivity of $100 \%, 98 \%$ and $92 \%$ respectively to predict hypoxemia. Difficulty in breathing had high specificity of $62.7 \%$ among the symptoms to predict hypoxemia in children with $\mathrm{ALRI}^{6,7,8}$. On comparison between hypoxemic and nonhypoxemic children difficulty in breathing and inability to feed had a $\mathrm{p}$ value $<0.001$ and was statistically significant.
Among signs, appearance of the child, lower chest retraction and tachypnoea had high sensitivity of $100 \%, 100 \%$ and $98 \%$ respectively and central cyanosis and grunting had specificity of $100 \%$ and $96.6 \%$ respectively to predict hypoxemia. None of symptoms and signs evaluated was both sufficiently sensitive and specific. Except for cyanosis, none of the clinical signs in children with lower respiratory tract infections can be explained by hypoxemia alone $^{9,10,11,12}$.

Table 2: Frequency of signs associated with hypoxemia

\begin{tabular}{|c|c|c|c|c|c|c|c|}
\hline Signs & $\begin{array}{c}\text { Hypoxemic } \\
\text { children }(50)\end{array}$ & $\begin{array}{l}\text { Non-hypoxemic } \\
\text { children (59) }\end{array}$ & Sensitivity & Specificity & Pvalue & PPV & NPV \\
\hline Appearance(sick look) & 50 & 41 & $100 \%$ & $30.5 \%$ & $<0.001$ & $55 \%$ & $100 \%$ \\
\hline Tachycardia & 31 & 25 & $62 \%$ & $57.6 \%$ & 0.041 & $64 \%$ & $55 \%$ \\
\hline Tachypnoea & 49 & 58 & $98 \%$ & $1.7 \%$ & 0.002 & $50 \%$ & $46 \%$ \\
\hline Cyanosis & 02 & 0 & $4 \%$ & $100 \%$ & 0.018 & $100 \%$ & $55 \%$ \\
\hline Nasalflaring & 29 & 13 & $58 \%$ & $78 \%$ & $<0.001$ & $69 \%$ & $68 \%$ \\
\hline Grunting & 17 & 02 & $34 \%$ & $96.6 \%$ & $<0.001$ & $89 \%$ & $63 \%$ \\
\hline Suprasternalindrawing & 32 & 12 & $64 \%$ & $79.7 \%$ & $<0.001$ & $73 \%$ & $72 \%$ \\
\hline Intercostalretractions & 41 & 17 & $82 \%$ & $71.2 \%$ & 0.7 & $71 \%$ & $82 \%$ \\
\hline Lower chestretractions & 50 & 58 & $100 \%$ & $1.7 \%$ & 0.275 & $46 \%$ & $100 \%$ \\
\hline Crepitations & 38 & 31 & $76 \%$ & $47.5 \%$ & 0.01 & $55 \%$ & $70 \%$ \\
\hline Decreased air entry & 39 & 41 & $78 \%$ & $31 \%$ & 0.4 & $49 \%$ & $62 \%$ \\
\hline
\end{tabular}

Among the signs, appearance, lower chest retraction, tachypnoea and intercostal retraction had high sensitivity of $100 \%$, $100 \%, 98 \%$ and $82 \%$ respectively to predict hypoxemia in children with ALRI. Cyanosis, grunting, suprasternal in drawing and nasal flaring had specificity of $100 \%$, $96.6 \%, 79.7 \%$ and $78 \%$ respectively to predict hypoxemia in children with ALRI. On comparison between hypoxemic and non-hypoxemic children $\mathrm{p}$ value was very highly significant in appearance, nasal flaring, grunting and suprasternal in drawing. Highly significant association was seen with tachypnoea and significant in tachycardia and crepitation ${ }^{13,14,15}$.

On combining the variables cough, inability to feed and tachypnoea showed highest sensitivity of $96 \%$ and combination of cough, tachypnoea, subcostal retractions had sensitivity of $95 \%$ followed by combination of cough, difficulty in breathing and tachypnoea which had a sensitivity of $90 \%$. Combination of 
Chinmay Sahu et.al. Original research article on study of correlation between various clinical parameters and hypoxemia in children with lower respiratory tract infection of age 2 months to 5 years

difficulty breathing, cyanosis and grunting had specificity of $98 \%$.

Table 3: Combination of variables to predict hypoxemia in children with ALRI

\begin{tabular}{|l|l|l|l|}
\hline Model & Combination Of variables & Sensitivity & Specificity \\
\hline Model-1 & Cough Difficulty inbreathing Tachypnoea & $90 \%$ & $11 \%$ \\
\hline Model-2 & Difficulty inbreathing Nasalflaring Crepitations & $82 \%$ & $15 \%$ \\
\hline Model-3 & Difficulty breathing Inability to feed Intercostalretractions & $80 \%$ & $13 \%$ \\
\hline Model-4 & Cough Inability to feed Tachypnoea & $96 \%$ & $20 \%$ \\
\hline Model-5 & CoughTachypnoea Intercostalretractions & $80 \%$ & $26 \%$ \\
\hline Model-6 & CoughTachypnoea Subcostalretractions & $95 \%$ & $10 \%$ \\
\hline Model-7 & Difficulty breathing Cyanosis Grunting & $36 \%$ & $98 \%$ \\
\hline
\end{tabular}

On combination of variables, model4 (cough, inability to feed, tachypnoea) and model-1 (cough, difficulty in breathing, tachypnoea) had high sensitivity of $96 \%$ and $90 \%$ to predict hypoxemia in children with ALRI. Model -2(difficulty in breathing, nasal flaring, crepitations), model3(difficulty in breathing, inability to feed, intercostal retraction) and model-5(cough, tachypnoea, intercostals retraction) had a sensitivity of $82 \%, 80 \%$ and $80 \%$ respectively to predict hypoxemia in children with ALRI. Model-6 (Cough, tachypnoea, subcostal retractions) had high sensitivity of $95 \%$ but low specificity of 10\%. Model-7 (Difficulty breathing, cyanosis, grunting) had highest specificity of $98 \%$.

\section{CONCLUSION}

The prevalence of hypoxemia in acute respiratory tract infection in this present study is $45.9 \%$.Correlation of symptoms and signs as a single indicator revealed inability to feed, cough, respiratory rate, sick looking appearance of the child had sensitivity of $100 \%, 98 \%, 92 \%, 100 \%$ respectively and cyanosis, grunting had specificity of $100 \%$ and $96.6 \%$ respectively; so have good ability to predict hypoxemia in acute lower respiratory tract infection. Combination of symptoms and signs further increase the sensitivity and specificity to predict hypoxemia. None of the clinical symptom or sign alone has both sufficient sensitivity and specificity to predict hypoxemia.

Acknowledgement: None

Conflict of Interest: None
Source of Funding: None

Ethical Approval: Approved

\section{REFERENCES}

1. Singhi S, Deepa A, Kaur H, Prevalence and predictors of hypoxemia in acute respiratory infections presenting in paediatric emergency department. Indian J Crit Care Med 2003;7:118-123.

2. Lodha R, Bhadauria PS, Kuttikat AV, Puranik M, Gupta S, Pandey RM, et al.Can clinical symptoms or signs accurately predict hypoxemia in children with acute lower respiratory tract infection? Indian Pediatr 2004;41:129-135.

3. Onyango FE, Steinoff MC, Wafula EM, Wariera, Musia J, Kitonia J. Hypoxemia in young Kenyan children with ALRI. BMJ 1993;306:612-614.

4. Weber MW, Usen S, Palmer A, Jaffar S, Mulholland EK. Predictors of hypoxemia in hospital admission with ALRI in developing countries. Arch Dis Child 1997;76:1-5.

5. Duke T, Blaschke AJ, Sialis S, Bonkowsky JL. Hypoxemia in acute respiratory and non-respiratory illness in neonates and children in developing countries. Arch Dis Child 2002;86:108-112.

6. J M Lozano, M Steinhoff, J G Ruiz, M L Mesa, N Martinez, B Dussan. Clinical predictors of acute radiological pneumonia and hypoxemia at high altitudes. Archives of Disease in Childhood 1994;71:323-327.

7. Sudha Basnet, Ramesh Kant Aadhikari and Chitra kumar Gurung. Hypoxemia in children with pneumonia and its clinical predictors. Indian J Pediatr 2006;73(9);777778.

8. Philip Ayeiko and Mike English. In children aged 2-59 months with pneumonia, which clinical signs best predict hypoxemia? J Trop Pediatr 2006;52(5):307-310.

9. Stanley Usen, Martin Weber, Kim Mulholland, Shabbar Jaffar. Clinical 
Chinmay Sahu et.al. Original research article on study of correlation between various clinical parameters and hypoxemia in children with lower respiratory tract infection of age 2 months to 5 years

predictors of hypoxemia in Gambian children with acute lower respiratory tract infection: prospective cohort study. BMJ 1999;318:86.

10. Onyango FE, Steinofff MC, Wafula EM, Wariera, Musia J, Kitonyi J. Hypoxemia in young Kenyan children with ALRI. BMJ 1993; 306:612- 614 .

11. Smyth A, Carty h, Hart CA.Clinical predictors of hypoxemia in children with pneumonia. Ann Trop Pediatr 1998;18 (1): 331-340.

12. Made Supartha, Putu SiadiPurniti, KoniNaning, Ida BagusSubanada. Clinical predictors of hypoxemia in 1-5 year old children with pneumonia. PediatricIndones. 2010;50:355-60.

13. Usen S, Weber MW. Clinical signs of hypoxemia in children with acute lower respiratory infection: Indicators of oxygen therapy. Int J Tuberc and lung dis. 2001; 5:505-10.
14. Muki K, Al- Janabi , Ridha H Al- Bayati, Nadia Aziz. Predictors of hypoxemia in children with acute lower respiratory tract infection. Iraqi Postgraduate Medical Journal 2009;8:40-46.

15. Yashwant Kumar Rao, Tanu Midha, Pankaj Kumar et al. Clinical predictors of hypoxemia in Indian children with acute respiratory tract infection presenting to pediatric emergency department. World J Pediatr. 2012 Aug;8(3):247-51. doi: 10.1007/s12519-012-0365-1.

How to cite this article: Sahu C, Panda DK, Naik $S$ et.al. Original research article on study of correlation between various clinical parameters and hypoxemia in children with lower respiratory tract infection of age 2 months to 5 years. International Journal of Research and Review. 2021; 8(9): 408-412. DOI: https://doi.org/10.52403/ijrr.20210952 